\title{
Proposta metodológica para levantamento e configuração de Densidade de Área Foliar (DAF) para aplicação no software ENVI-met
}

\section{Methodological proposal for the assessment and configuration of Leaf Area Density (LAD) for application in ENVI-met software}

\author{
${ }^{1}$ Karyna de A. Carvalho Rosseti, ${ }^{2}$ Malu Herrera Scaffi, ${ }^{3}$ Ivan Julio Apolônio Callejas, ${ }^{4}$ Luciane Cleonice Durante \\ ${ }^{1}$ Prof. a Doutora em Física Ambiental - Universidade Federal de Mato Grosso (karyna.rosseti@gmail.com) \\ ${ }^{2}$ Arquiteta - Graduada pela Universidade Federal de Mato Grosso (maluscaffi94@ gmail.com) \\ ${ }^{3}$ Prof. Doutor em Física Ambiental - Universidade Federal de Mato Grosso (ivancallejas1973@ gmail.com)

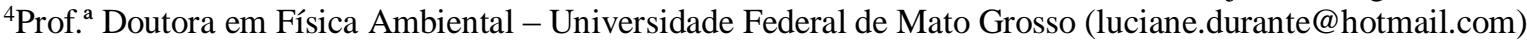

\begin{abstract}
RESUMO: Atualmente, ferramentas computacionais vêm sendo utilizadas para fins de investigar os efeitos da incorporação de indivíduos arbóreos nas características microclimáticas dos ambientes externos. O software ENVI-met é um modelo tridimensional não hidrostático, que simula as interações entre as superfícies urbanizadas, vegetação e atmosfera. Esta ferramenta computacional possui uma biblioteca de vegetação desenvolvida com base em estruturas vegetativas encontradas em climas temperados, as quais não são capazes de descrever adequadamente vegetação existente em climas tropicais. Objetiva-se nesta pesquisa estabelecer uma metodologia de levantamento e configuração de Densidade de Área Foliar (DAF) visando a sua incorporação em banco de dados de vegetação do software ENVI-met. Utilizou-se uma técnica de fotografias hemisféricas, adaptada para ser usada por meio do uso de Smatphones. Por meio de levantamento de campo, o primeiro passo metodológico foi a identificação de padrões de densidade de área foliar e geometria de copa das árvores de elevada frequência de ocorrência em cidade de clima tropical em região central do Brasil. Na etapa seguinte, identificou-se o Índice de Área Foliar das espécies selecionadas, por meio da análise de fotografias hemisféricas no software Gap Light Analyzer (GLA), com posterior validação por meio do Equipamento denominado de Ceptômetro. Na sequencia, configurou-se a biblioteca de vegetação do software ENVI-Met. Os resultados possibilitaram a consolidação da metodologia de levantamento com uso de ferramentas simples e acessíveis, além da criação de um banco de dados de vegetação para subsidiar trabalhos futuros que sejam desenvolvidos em regiões de clima tropical.
\end{abstract}

Palavras Chave: Fotografia hemisférica. Modelagem computacional. Clima urbano.

\begin{abstract}
Computational tools have been used to investigate the effects of the arboreal individual's incorporation into the characteristics of outdoor microclimatic environment. The ENVI-met software is a nonhydrostatic three-dimensional model that simulates the interactions between urbanized surfaces, vegetation and atmosphere. This computational tool has a vegetation library based on vegetative structures developed for temperate climates which are not able to describe adequately the existing vegetation in tropical climates. The aim of this research is to establish a methodology to access and to configure the Leaf Area Density (LAD) focusing to incorporate it into the ENVI-met software vegetation database. A hemispherical photographs technique was used, adapted to be used by the use of Smatphones. Through field survey, the first step was to identify the leaf area density and crown geometry of most frequency trees existing in a tropical climate city in Brazil's Midwest. In the next step, the selected species's Leaf Area Index were identified, through the analysis of hemispheric photographs in the Gap Light Analyzer (GLA) software, with further validation by the use of Ceptômetro equipment. In sequence, the ENVI-Met software vegetation database was set up. The results allowed the consolidation of the survey methodology with the use of simple and accessible tools, as well as the creation of a vegetation database to support future works that will be developed in tropical climates.
\end{abstract}

Keywords: Hemispheric photography. Computational modeling. Urban climate.

\section{INTRODUÇÃO}

Dentre as principais consequências das modificações do ambiente natural causadas pelo processo de urbanização, tem-se o fenômeno de ilha de calor urbano (ICU), caracterizado pelo aumento da temperatura média das áreas urbanas em relação às de seu entorno não urbanizadas (OKE, 1982). Muitos fatores contribuem para o fenômeno, tais como a supressão das áreas verdes, que reduz as trocas térmicas por evapotranspiração e, a impermeabilização 
do solo, que faz com que a maior parte da energia disponível no meio durante o dia devido a entrada da radiação solar seja trocada na forma de calor sensível ou estocada dentro dos materiais que compõem a cidade, ocasionando grandes variações térmicas diárias, tanto nas superfícies quanto no ar do dossel urbano (OKE, 1982; HUANG et al., 2009).

Estudos tem apontado a importância da recuperação da vegetação para ambiência urbana, demonstrando o potencial de minimização dos efeitos das ICU e melhoria da qualidade de vida da população (NAKATA-OSAKI, et al., 2016; HONJO; TAKAKURA, 1990-1991; UPMANIS et al., 1998, AKBARI et al., 2001). No entanto, ainda existe uma lacuna quanto a alguns parâmetros, como a definição dos melhores espécimes e a adequada disposição da vegetação nos projetos urbanísticos, com vista a obter resultados mais eficientes sob o ponto de vista do conforto ambiental de áreas externas.

Uma das alternativas para demonstrar os potenciais benefícios da vegetação aos ambientes externos urbanos é por meio de simulação computacional. Entre os vários softwares disponíveis, tem-se o ENVI-met, um programa capaz de modelar numericamente e tridimensionalmente microclimas urbanos, a partir da consideração da interação entre solo, água e atmosfera (BRUSE; FLEER, 1998). Em virtude de sua versatilidade, várias pesquisas têm sido conduzidas visando delinear o comportamento da vegetação quando inseridas nos ambientes externos urbanos (MARCONDES e UMAKOSHI, 2007, NAKATA, 2010), ROMERO, 2011, ROSSETI et al., 2015) e SPANGENBERG et al., 2019). Apesar dos benefícios evidenciados nessas pesquisas, identificou-se que foram conduzidas a partir de um banco de dados de vegetação elaborado com base em estruturas vegetativas encontradas em climas temperados, não sendo capazes, desta forma, de descrever adequadamente a vegetação presente em clima tropical.

Uma das configurações necessárias para a simulação computacional no ENVI-met é destinada a representar os espécimes arbóreos existentes na área de estudo. A representação da vegetação é feita por meio do ajuste da Densidade de Área Foliar (DAF) existente em cada camada da copa da árvore, indo da superfície do solo até o topo da copa. A DAF é definida como a área foliar unilateral total por unidade de volume da copa $\left(\mathrm{m}^{2} / \mathrm{m}^{3}\right)$ (HOSOI; OMASA, 2009). Este parâmetro permite definir não somente a densidade de folhagens da copa, mas também o seu formato. O perfil de DAF pode ser definido por meio de mediação da quantidade de área de folhas em um determinado volume ou, ainda, derivado de outros parâmetros, como, por exemplo, a partir do Índice de Área Foliar (IAF), um parâmetro biofísico adimensional da vegetação definido como a área foliar integrada do dossel por unidade de superfície projetada no solo $\left(\mathrm{m}^{2} / \mathrm{m}^{2}\right)$ (WATSON, 1952).

O levantamento do IAF pode ser realizado por meio de métodos diretos, também conhecidos por métodos "destrutivos", considerados padrões por diversos autores (FAVARIN et al., 2002). Pode-se, ainda, realizar o levantamento do IAF por meio de métodos indiretos, que realizam estimativas do IAF mantendo a integridade das folhas das árvores, sem a necessidade de subtração da copa.

A utilização de fotos hemisféricas para quantificar o IAF é um método indireto, não destrutível, pois permite descrever a abertura de dossel e relações de luz, que por meio de softwares específicos, possibilitam a obtenção de valores de IAF (NASCIMENTO, 2007). As fotos hemisféricas utilizadas para estudar as copas das árvores podem ser obtidas por meio de uma máquina fotográfica digital com a lente situada sob a copa (orientada para Zênite) ou sobre a copa com a lente voltada para baixo. No entanto, a metodologia envolve um alto investimento na aquisição de equipamento fotográfico e, ainda, de acessórios, como a lente olho-de-peixe (AMORIM, 2014).

Esta pesquisa foca na possiblidade de utilização de equipamentos alternativos para a medição de IAF das copas das árvores, com vistas a derivar parâmetros que possam ser incorporados para a formação de um banco de dados de espécimes arbóreos de região de 
clima tropical, para utilização em simulações computacionais. Assim, este trabalho tem por objetivo estabelecer uma metodologia de levantamento e configuração de Densidade de Área Foliar (DAF), com uso de técnica alternativa de fotografias hemisféricas por meio de Smatphones, visando a adequação do banco de dados de vegetação do software de simulação computacional ENVI-met para aplicação em regiões de clima tropical.

\section{MATERIAIS E MÉTODOS}

\subsection{Local da Pesquisa}

De forma a possibilitar a seleção de vegetação característica de clima tropical, foi selecionado para realização da pesquisa o município de Cuiabá, Mato Grosso, Brasil $\left(15^{\circ} 35^{\prime}\right.$ $56^{\prime \prime} \mathrm{S}, 56^{\circ} 06^{\prime} 01^{\prime \prime} \mathrm{W}$ ). A cidade possui área de 254,57 km² (CUIABÁ, 2010) e se insere em região clima tropical semiúmido (Aw), caracterizado por precipitação elevada na primaveraverão e por seca acentuada no outono-inverno (MACHADO, et al. 2015). A vegetação predominante na região é o Cerrado, sendo que as matas mais densas são encontradas nas proximidades dos cursos d'água. Na cidade, a vegetação é encontrada, principalmente, em praças, nos domicílios e ao longo da malha viária.

\subsection{Definição das categorias de Vegetação e geometria das copas}

A estrutura vertical de folhagem de indivíduos arbóreos, usualmente, é calculada por meio do parâmetro de densidade da área foliar (DAF) em cada camada da copa da árvore (indo desde a superfície do solo até o topo da copa). Visando delinear esta característica da copa, definiram-se três padrões de densidade foliar entre as espécies arbóreas mais comumente encontradas na arborização pública na cidade, considerando tanto espécies nativas quanto exóticas. Assim, as espécies foram agrupadas, conforme padrão visual de permeabilidade da copa nas categorias de "baixa densidade", "média densidade" e "alta densidade". É importante destacar que este agrupamento deve ser realizado por uma única pessoa de forma a evitar conflitos de interpretação.

Para definição do fator de distribuição da DAF em cada camada da copa da árvore foram observados os padrões geométricos das árvores selecionadas, conforme proposto por Shinzato (2009) (Figura 1), assumindo-se a homogeneidade da DAF no interior das camadas. Buscou-se selecionar árvores com semelhança na geometria da copa, de forma a minimizar a influência das variações desta geometria na distribuição da DAF.

O modelo ENVI-met, em seu banco de dados de vegetação, caracteriza a DAF por meio da divisão da altura do indivíduo arbóreo em dez camadas, contadas acima do solo até o topo da copa. A somatória da DAF de cada uma das camadas conduz ao valor do IAF da vegetação.

Figura 1 - (a) Exemplo de geometrias de copas arredondada e alongada, (b) fatores de distribuição da DAF, em 10 camadas, obtido para copas arredondadas e (c) fatores de distribuição da DAF para copas alongadas

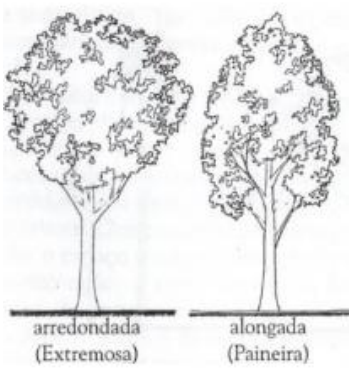

(a)
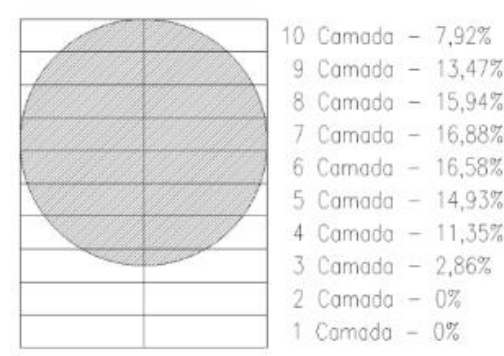

(b)
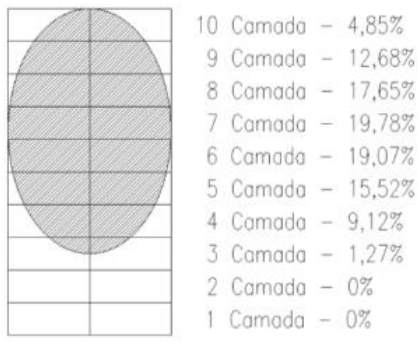

(c)

Fonte: Gusson e Shinzato (2015). 
Rosseti, Karyna, et al.; Proposta metodológica para levantamento e configuração de Densidade de Área Foliar (DAF) para aplicação no software ENVI-met. E\&S - Engineering and Science, 2019, 8:2.

\subsection{Levantamento de campo com Fotografia Hemisférica e classificação manual do IAF por meio de software}

Para a definição do horário de obtenção das fotos, seguiu-se a recomendação de Souza e Ponzoni (1998), que indica que a coleta dos dados deve ser realizada com céu nublado (radiação difusa) para que haja pouco espalhamento da radiação eletromagnética (REM) pela folhagem, evitando uma possível subestimativa do IAF. Em dias de céu limpo, deve-se utilizar o equipamento no início do dia ou no final da tarde, quando o ângulo de elevação solar é baixo conforme recomendação dos autores.

Realizaram-se testes em três diferentes horários do dia para verificar os efeitos descritos por Souza e Ponzoni (1998) (Figura 2), nos quais confirmou-se a maior adequação de aplicação da metodologia nos horários de nascer e pôr do sol. Optou-se por trabalhar somente com o horário do nascer do sol para evitar transtornos e dados incoerentes provocados pelo repentino acionamento da iluminação pública.

Figura 2 - (a) Fotografias obtidas por meio da olho-de-peixe logo após o nascer do sol, (b) ao meio dia e (c) um pouco cantes do pôr do sol, para validação do melhor horário de levantamento

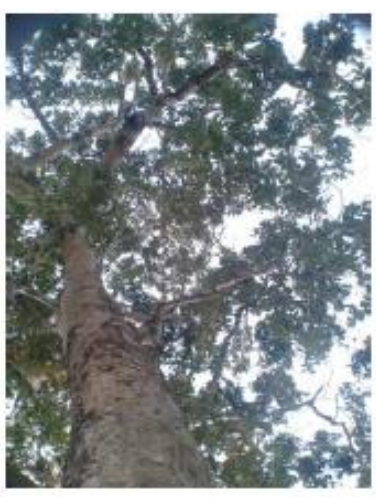

(a)

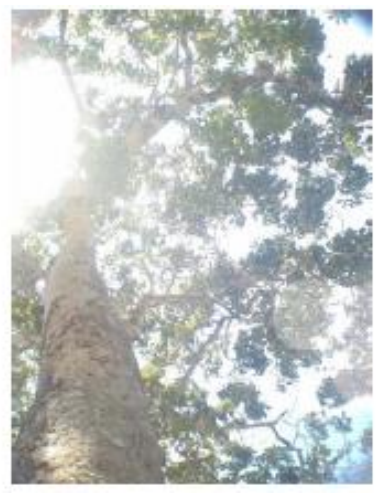

(b)

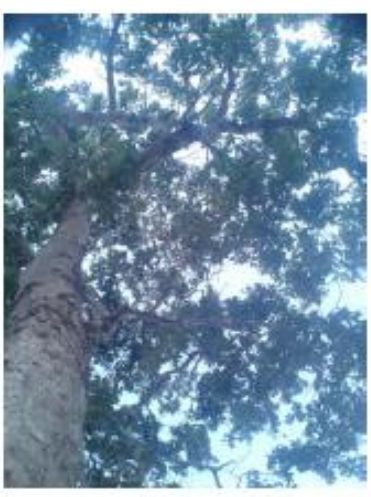

(c)

O levantamento fotográfico foi realizado no período seco considerando as condições de céu mais favoráveis para o levantamento. Foram selecionadas três árvores em cada categoria de densidade de área foliar, a fim de se obter a média do IAF de cada uma das categorias. Como as árvores deveriam estar isoladas (sem interferência de outras espécies ou mesmos elementos edificados) não foi possível encontrar representantes de todas as categorias de densidade foliar em um mesmo local.

Para garantir o mínimo de interferência da movimentação do sol na abóbada celeste durante cada sequência, buscou-se a maior proximidade possível dos indivíduos arbóreos selecionados em cada categoria de densidade. Neste sentido, foi necessário utilizar três diferentes locais para a realização das medições, todos dentro do perímetro urbano da cidade conforme indicado na Figura 3. O primeiro levantamento (baixa densidade) foi conduzido no campus da Universidade Federal de Mato Grosso, enquanto o segundo (média densidade), no bairro Jardim Califórnia, e o último (alta densidade), no bairro Condomínio Florais Cuiabá. 
Rosseti, Karyna, et al.; Proposta metodológica para levantamento e configuração de Densidade de Área Foliar (DAF) para aplicação no software ENVI-met. E\&S - Engineering and Science, 2019, 8:2.

Figura 3 - Imagens de satélite com identificação do (a) condomínio Florais Cuiabá, (b) Campus da Universidade Federal de Mato Grosso e (c) bairro Jardim Califórnia
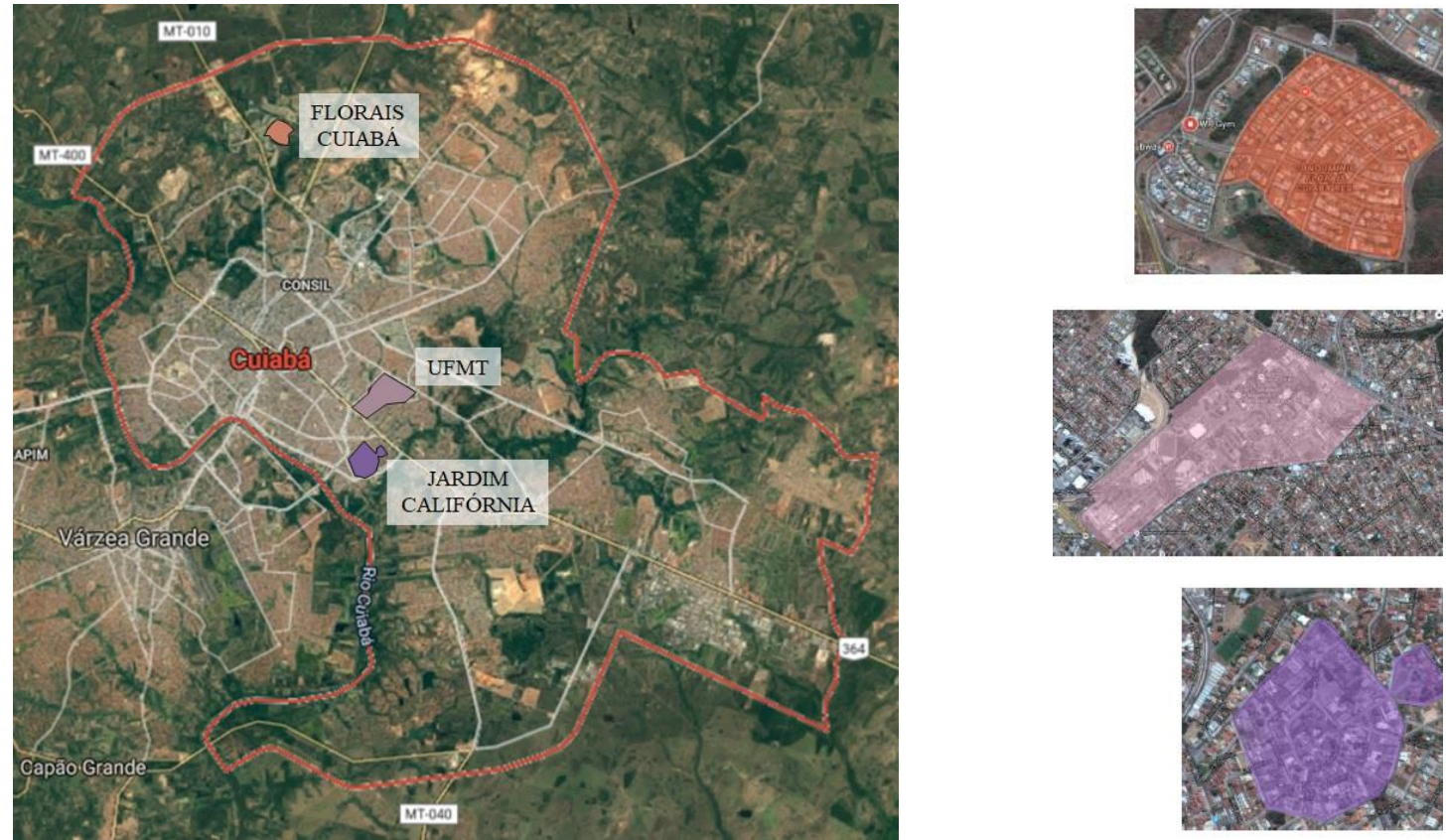

(a)

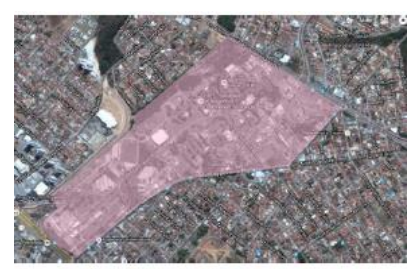

(b)

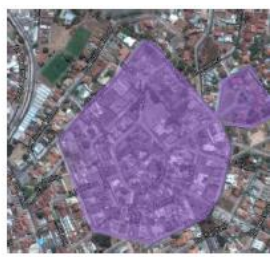

(c)

Utilizou-se o método de fotografias hemisféricas para obtenção do IAF, por meio do uso de uma lente olho-de-peixe acoplada a um dispositivo móvel (smartphone), conforme proposto por Amorim (2014) e Miranda et. al. (2018). A lente foi acoplada ao smartphone, que foi fixado a um tripé para realizar o ajuste de nível e altura da câmera em relação ao chão, de forma a garantir a padronização do levantamento. $\mathrm{Na}$ figura 4, ilustram-se objetos utilizados no processo de confecção das fotografias hemisféricas.

Figura 4 - Lente olho-de-peixe para acoplar em aparelho smartphone (a) e Tripé com base ajustável (b)

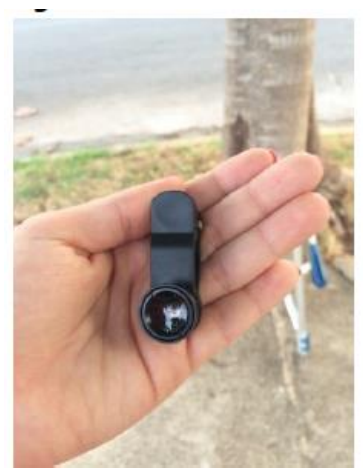

(a)

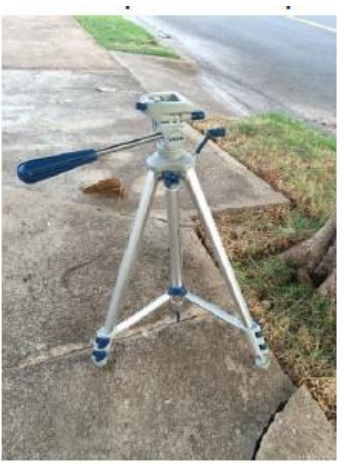

(b)

As fotografias foram analisadas com auxílio do software Gap Light Analyzer (GLA (C)1999, Simon Fraser University, Burnaby, British Columbia, Canadá), visando à obtenção do índice de área foliar para cada árvore fotografada nas três regiões definidas para o levantamento.

Trabalhou-se tanto com a técnica de seleção da imagem quanto com os parâmetros de configuração do software visando obter uma adequada quantificação do IAF. Optou-se por trabalhar com duas abrangências de seleção: a primeira considerando uma pequena porção homogênea da imagem captada e, outra, com seleção de toda a porção da imagem da árvore, considerando regiões de tronco e folhas. Cabe salientar a necessidade de condução do 
Rosseti, Karyna, et al.; Proposta metodológica para levantamento e configuração de Densidade de Área Foliar (DAF) para aplicação no software ENVI-met. E\&S - Engineering and Science, 2019, 8:2.

processamento no software por uma única pessoa, com intuito de evitar diferentes interpretações durante o manuseio das imagens.

\subsection{Validação do método fotografias hemisféricas com lente de olho-de-peixe}

Para a validação do método da fotografia hemisférica (lente olho-de-peixe) foi utilizado o equipamento denominado de ceptômetro de barra, modelo LP-80 AccuPAR, Decagron Devices, um aparelho que mede a Radiação Fotossinteticamente Ativa (RFA). As medições foram feitas imediatamente próximas à copa e em quatro pontos sob a copa, para se determinar a interceptação da luz em cinco ângulos, sendo o IAF gerado por meio de modelos de transferência de radiação nas copas das árvores.

A fim de obter valores mais coerentes com as medições destrutivas, deve-se medir com o ceptômetro no horário em que o ângulo solar vertical está próximo a $90^{\circ}$ (das 11 às 14h) e, ainda, possuir condições de céu limpo. Tais condições somente foram encontradas na pesquisa no período seco.

Os dados obtidos no levantamento com ceptômetro foram correlacionados com os obtidos na análise das fotografias hemisféricas no software Gap Light Analyzer visando estabelecer relação entre os dados derivados por meio das duas metodologias de levantamento. Para aferir o grau de correlação entre estes dados, adotou-se o coeficiente de determinação $\left(\mathrm{R}^{2}\right)$, que indica uma medida de ajustamento de um modelo estatístico linear generalizado. Destaca-se que quanto maior o $\mathrm{R}^{2}$, mais explicativo é o modelo, ou seja, melhor ele se ajusta à amostra.

\section{RESULTADOS/ DISCUSSÕES}

\subsection{Fator de Distribuição da DAF}

O perfil geométrico de todas as árvores selecionadas foi analisado, conforme proposto por Shinzato (2009) (Figura 5) e os fatores de distribuição da DAF nas dez camadas da copa podem ser visualizados na Tabela 1.

Figura 5 - Perfil geométrico das árvores selecionadas e distribuição da área de copa em dez camadas, contadas a partir do solo ao topo da copa
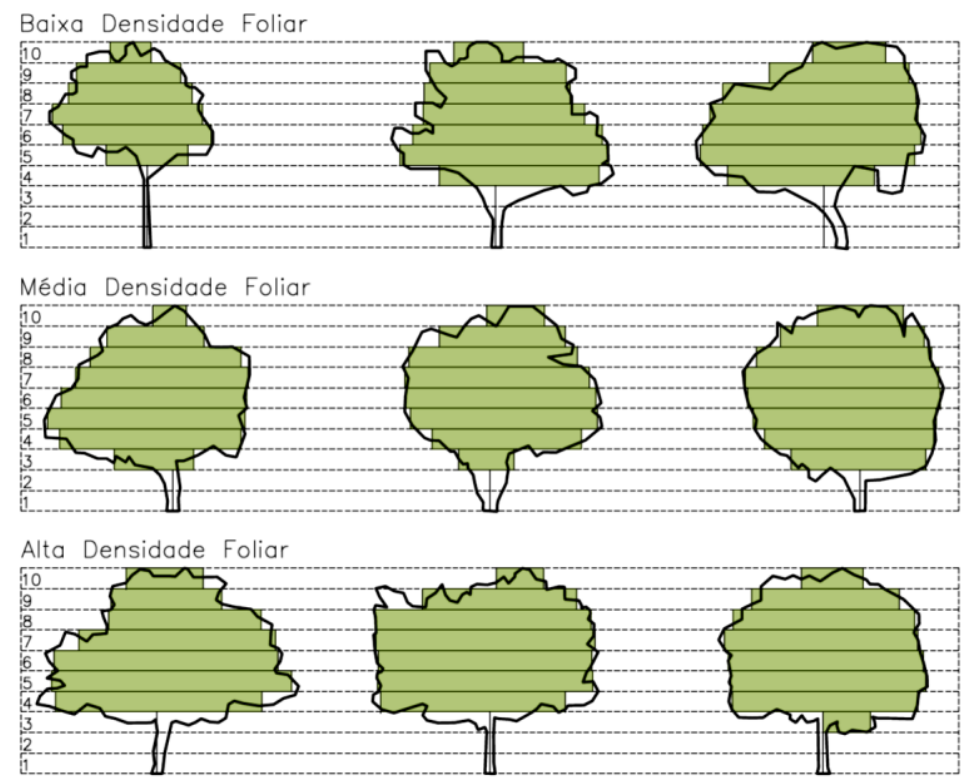
Rosseti, Karyna, et al.; Proposta metodológica para levantamento e configuração de Densidade de Área Foliar (DAF) para aplicação no software ENVI-met. E\&S - Engineering and Science, 2019, 8:2.

Tabela 1 - Definição do Fator de DAF para as 10 camadas da copa, considerando as 3 categorias de densidade analisadas no estudo

\begin{tabular}{|c|c|c|c|c|c|c|c|c|c|c|c|c|c|c|}
\hline \multirow{4}{*}{ שֶّ } & \multicolumn{14}{|c|}{ Fator DAF } \\
\hline & \multicolumn{4}{|c|}{ Baixa Densidade } & \multicolumn{4}{|c|}{ Média Densidade } & \multicolumn{4}{|c|}{ Alta Densidade } & \multirow{3}{*}{ 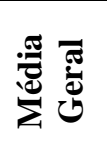 } & \multirow{3}{*}{ } \\
\hline & \multicolumn{3}{|c|}{ Árvores } & \multirow{2}{*}{ Média } & \multicolumn{3}{|c|}{ Árvores } & \multirow{2}{*}{ Média } & \multicolumn{3}{|c|}{ Árvores } & \multirow{2}{*}{ Média } & & \\
\hline & 1 & 2 & 3 & & 1 & 2 & 3 & & 1 & 2 & 3 & & & \\
\hline 10 & $6 \%$ & $6 \%$ & $6 \%$ & $6 \%$ & $3 \%$ & $5 \%$ & $8 \%$ & $5 \%$ & $6 \%$ & $4 \%$ & $6 \%$ & $5 \%$ & $5,5 \%$ & $0,4 \%$ \\
\hline 9 & $16 \%$ & $13 \%$ & $12 \%$ & $14 \%$ & $9 \%$ & $11 \%$ & $11 \%$ & $10 \%$ & $9 \%$ & $12 \%$ & $12 \%$ & $11 \%$ & $11,7 \%$ & $1,8 \%$ \\
\hline 8 & $19 \%$ & $14 \%$ & $16 \%$ & $16 \%$ & $14 \%$ & $15 \%$ & $14 \%$ & $14 \%$ & $14 \%$ & $17 \%$ & $15 \%$ & $15 \%$ & $15,3 \%$ & $1,0 \%$ \\
\hline 7 & $23 \%$ & $15 \%$ & $17 \%$ & $18 \%$ & $16 \%$ & $16 \%$ & $15 \%$ & $16 \%$ & $16 \%$ & $18 \%$ & $16 \%$ & $17 \%$ & $16,9 \%$ & $1,3 \%$ \\
\hline 6 & $23 \%$ & $18 \%$ & $19 \%$ & $20 \%$ & $17 \%$ & $18 \%$ & $15 \%$ & $17 \%$ & $18 \%$ & $17 \%$ & $16 \%$ & $17 \%$ & $17,9 \%$ & $1,8 \%$ \\
\hline 5 & $13 \%$ & $19 \%$ & $18 \%$ & $17 \%$ & $18 \%$ & $17 \%$ & $14 \%$ & $16 \%$ & $20 \%$ & $17 \%$ & $16 \%$ & $18 \%$ & $16,9 \%$ & $0,7 \%$ \\
\hline 4 & $0 \%$ & $15 \%$ & $12 \%$ & $9 \%$ & $17 \%$ & $13 \%$ & $13 \%$ & $14 \%$ & $17 \%$ & $15 \%$ & $15 \%$ & $16 \%$ & $13,0 \%$ & $3,5 \%$ \\
\hline 3 & $0 \%$ & $0 \%$ & $0 \%$ & $0 \%$ & $6 \%$ & $5 \%$ & $10 \%$ & $7 \%$ & $0 \%$ & $0 \%$ & $4 \%$ & $1 \%$ & $2,8 \%$ & $3,7 \%$ \\
\hline 2 & $0 \%$ & $0 \%$ & $0 \%$ & $0 \%$ & $0 \%$ & $0 \%$ & $0 \%$ & $0 \%$ & $0 \%$ & $0 \%$ & $0 \%$ & $0 \%$ & $0,0 \%$ & $0,0 \%$ \\
\hline 1 & $0 \%$ & $0 \%$ & $0 \%$ & $0 \%$ & $0 \%$ & $0 \%$ & $0 \%$ & $0 \%$ & $0 \%$ & $0 \%$ & $0 \%$ & $0 \%$ & $0,0 \%$ & $0,0 \%$ \\
\hline \multicolumn{5}{|c|}{$100 \%$} & \multicolumn{4}{|c|}{$100 \%$} & \multicolumn{4}{|c|}{$100 \%$} & & \\
\hline
\end{tabular}

\section{2 Índices de Área Foliar}

Os resultados obtidos por meio da metodologia de fotografias hemisféricas e por meio do ceptômetro podem ser visualizados na Tabela 2.

Tabela 2 - IAF para as categorias de baixa, média e alta densidade de área foliar obtidos por meio da metodologia de fotografia hemisférica (com seleção de menor e maior porção da imagem) e ceptômetro

\begin{tabular}{|c|c|c|c|c|}
\hline \multirow[b]{2}{*}{ Levantamento } & \multirow[b]{2}{*}{ Sequência } & \multicolumn{3}{|c|}{ IAF } \\
\hline & & $\begin{array}{c}\text { Baixa } \\
\text { Densidade }\end{array}$ & $\begin{array}{c}\text { Média } \\
\text { Densidade }\end{array}$ & $\begin{array}{c}\text { Alta } \\
\text { Densidade }\end{array}$ \\
\hline \multirow{3}{*}{$\begin{array}{c}\text { GLA Menor } \\
\text { Porção }\end{array}$} & Árvore 01 & 2,28 & 3,83 & 3,91 \\
\hline & Árvore 02 & 2,06 & 3,76 & 5,4 \\
\hline & Árvore 03 & 2,82 & 4,07 & 6,41 \\
\hline \multirow{3}{*}{$\begin{array}{l}\text { GLA Maior } \\
\text { Porção }\end{array}$} & Árvore 01 & 2,44 & 3,46 & 4,10 \\
\hline & Árvore 02 & 2,41 & 3,49 & 4,98 \\
\hline & Árvore 03 & 3,25 & 3,80 & 5,20 \\
\hline \multirow{3}{*}{ Ceptômetro } & Árvore 01 & 0,26 & 3,98 & 5,23 \\
\hline & Árvore 02 & 3,82 & 5,04 & 7,12 \\
\hline & Árvore 03 & 2,84 & 5,28 & 7,18 \\
\hline
\end{tabular}

Os dados de IAF medidos com o ceptômetro e os levantados com a fotografia hemisférica foram analisados comparativamente por meio de diagramas de dispersão e dos coeficientes de correlação linear, para cada categoria de árvore de acordo com a sua densidade. Foram gerados diagramas de dispersão (Figura 6) considerando as duas técnicas de cálculo utilizadas no software GLA (menor e maior porção da imagem). 
Rosseti, Karyna, et al.; Proposta metodológica para levantamento e configuração de Densidade de Área Foliar (DAF) para aplicação no software ENVI-met. E\&S - Engineering and Science, 2019, 8:2.

Figura 6 - Correlação entre os valores obtidos por meio de ceptômetro com (a) IAF calculado pelo GLA para menor porção da imagem e (b) com IAF calculado pelo GLA para maior porção da imagem

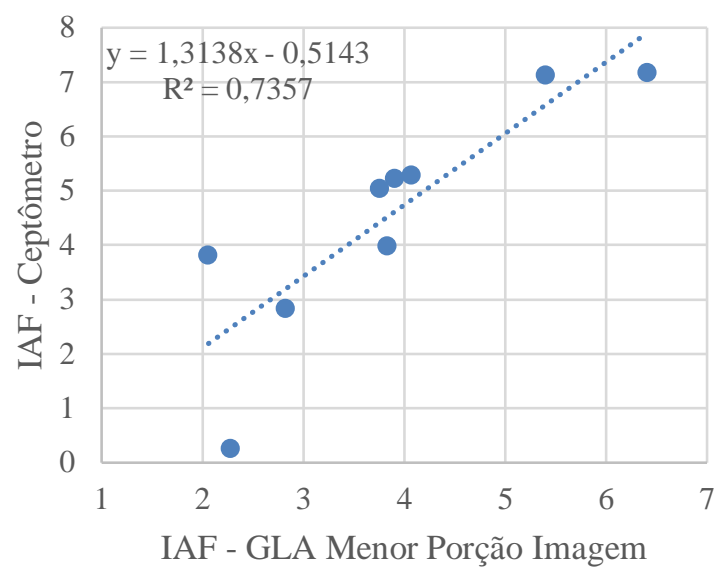

(a)

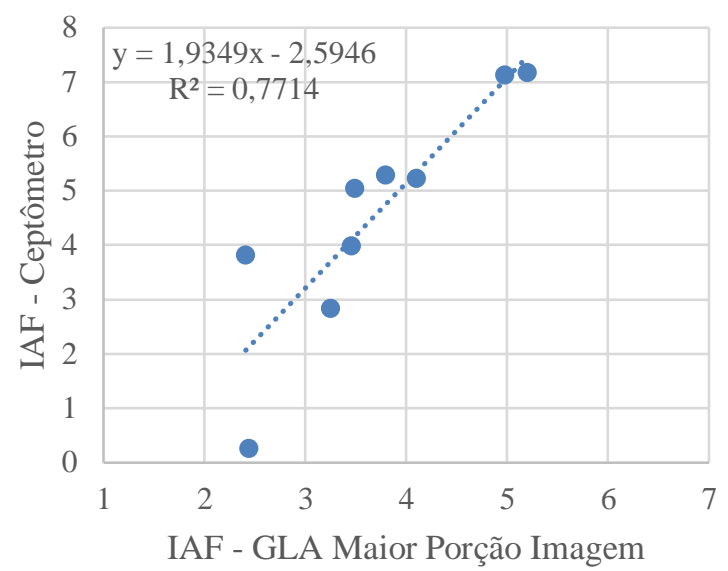

(b)

Observa-se na Figura 6 que, a técnica de cálculo da fotografia hemisférica por meio da seleção de menor e maior porção da imagem apresentou correlação positiva com os dados obtidos por meio do ceptômetro $(0,74$ e 0,77 , respectivamente). As duas correlações são elevadas, indicando que é possível derivar os valores obtidos por meio de ceptômetro com adequada precisão a partir de fotografias hemisféricas.

Observou-se que ocorreram discrepâncias entre os resultados obtidos por meio das duas metodologias de levantamento para a árvore denominada 01, com característica de baixa densidade. Usando a técnica de cálculo com seleção de menor e maior porção da imagem obteve-se, respectivamente, IAF de 2,28 e $2,44 \mathrm{~m}^{2} / \mathrm{m}^{2}$, enquanto que, para o levantamento com o ceptômetro, por repetidas vezes, a árvore 01 apresentou resultados menores que os das fotos hemisféricas, com IAF de $0,26 \mathrm{~m}^{2} / \mathrm{m}^{2}$. Atribui-se essa diferença à altura da árvore 01 , uma vez que esta apresentava altura elevada, fazendo com que sua copa barrasse pouca radiação no momento do levantamento com o ceptômetro. A altura não é significativa no levantamento com a fotografia hemisférica, uma vez que por meio do software Gap Light Analyzer é possível calcular o IAF com base na seleção da região específica de copa. Portanto, optou-se por realizar nova análise desconsiderando o levantamento conduzido na Árvore 01, conforme indicado na Figura 7.

Figura 7 - Correlação entre os valores obtidos por meio de ceptômetro com (a) IAF calculado pelo GLA para menor porção da imagem e (b) com IAF calculado pelo GLA para maior porção da imagem

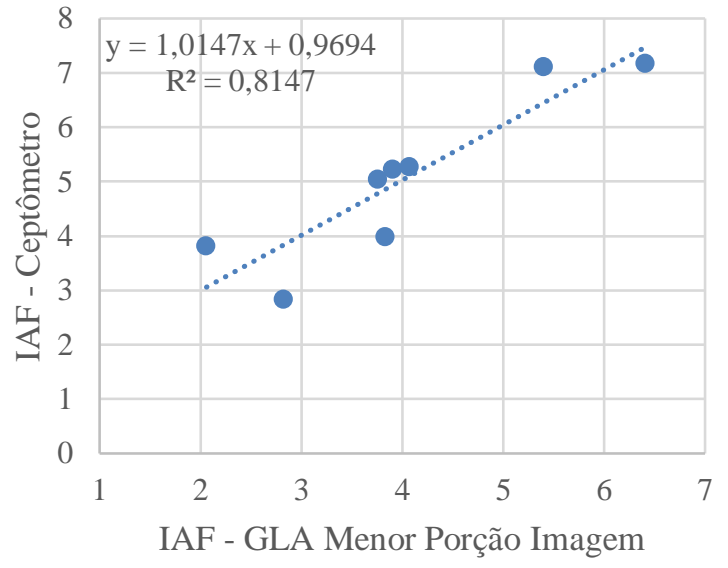

(a)

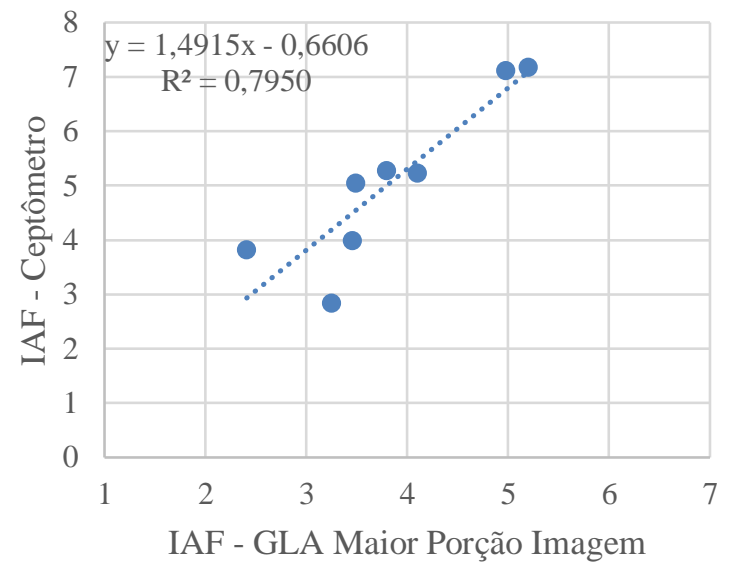

(b) 
Observa-se na Figura 7, que tanto a técnica de cálculo da fotografia hemisférica por meio da seleção de pequena porção da imagem quanto a de maior porção da imagem apresentaram correlação positiva com os dados obtidos por meio do ceptômetro. Destaca-se que, dentre as duas metodologias, com a retirada da Árvore 01 de baixa densidade da amostra, a com seleção de menor porção da imagem apresentou maior coeficiente de determinação $(0,81)$ do que a com seleção de maior porção da imagem $(0,79)$. Ao se conduzir uma avaliação do erro cometido ao se aplicar a equação determinada na Figura 7a, chega-se a um erro médio absoluto de $0,54 \mathrm{~m}^{2} / \mathrm{m}^{2}$, enquanto para a determinada na Figura $7 \mathrm{~b}$, de $0,52 \mathrm{~m}^{2} / \mathrm{m}^{2}$, o que representa um erro médio de $10,5 \%$ e $9,7 \%$, respectivamente, em relação ao valor médio determinado por meio do ceptômetro.

Para fins de calibração dos dados para determinação da DAF optou-se por utilizar a equação de regressão linear com maior coeficiente de determinação $\left(\mathrm{R}^{2}\right)$ obtida a partir da correlação do levantamento de fotografias hemisféricas com seleção de menor porção da imagem com o uso do ceptômetro (Equação 1, sendo y o valor de IAF corrigido e x o valor medido por meio das fotografias hemisféricas com seleção de menor porção da imagem).

$$
I A F_{C E P}=1,0147 \cdot I A F_{G L A}+0,9694
$$

Os valores finais de Índice de área foliar, obtidos por meio média calculada pelo método de fotografia hemisférica, com seleção de menor porção da imagem, desprezando-se a árvore 01 de baixa densidade, com posterior ajuste apresentado na Equação 1, foram $3,445 \mathrm{~m}^{2} / \mathrm{m}^{2}$ para árvores de baixa densidade, $4,913 \mathrm{~m}^{2} / \mathrm{m}^{2}$ para as de média densidade e $6,286 \mathrm{~m}^{2} / \mathrm{m}^{2}$ para as de alta densidade.

Reforça-se que caso a metodologia seja aplicada a padrões de geometria e densidade foliar diferenciados dos utilizados neste estudo, deve-se proceder com a metodologia de calibração por meio do ceptômetro para obter a equação de ajuste adequada para a vegetação selecionada.

\subsection{Perfil de Densidade de Área Foliar}

Como relatado, o software ENVI-met considerada a variável densidade de área foliar (DAF) para caracterização de seu banco de dados de vegetação. Portanto, foi preciso transformar a variável Índice de Área Foliar - IAF em DAF. Os resultados foram obtidos relacionando a média do IAF de cada categoria de densidade com os fatores de distribuição do IAF nas camadas da árvore conforme descrito no item 3.1 (Tabela 3).

Tabela 3 - DAF para cada porção de volume da vegetação, contadas do topo da árvore (camada 10) à superfície do solo (camada 1), conforme proporção geométrica descrita na Figura 04

\begin{tabular}{ccccc}
\hline \multirow{2}{*}{ Camada } & Fator & $\begin{array}{c}\text { DAF } \\
\text { Baixa } \\
\text { Densidade }\end{array}$ & $\begin{array}{c}\text { Média } \\
\text { Densidade }\end{array}$ & $\begin{array}{c}\text { Alta } \\
\text { Densidade }\end{array}$ \\
\hline 10 & $5,5 \%$ & 0,189 & 0,270 & 0,346 \\
\hline 9 & $11,7 \%$ & 0,403 & 0,575 & 0,736 \\
\hline 8 & $15,3 \%$ & 0,527 & 0,752 & 0,962 \\
\hline 7 & $16,9 \%$ & 0,582 & 0,830 & 1,062 \\
\hline 6 & $17,9 \%$ & 0,617 & 0,879 & 1,125 \\
\hline 5 & $16,9 \%$ & 0,582 & 0,830 & 1,062 \\
\hline 4 & $13,0 \%$ & 0,448 & 0,639 & 0,817 \\
\hline 3 & $2,8 \%$ & 0,096 & 0,138 & 0,176 \\
\hline 2 & $0,0 \%$ & 0,000 & 0,000 & 0,000 \\
\hline 1 & $0,0 \%$ & 0,000 & 0,000 & 0,000 \\
\hline SOMA & $\mathbf{1 0 0 \%}$ & 3,445 & 4,913 & 6,286 \\
\hline
\end{tabular}


Rosseti, Karyna, et al.; Proposta metodológica para levantamento e configuração de Densidade de Área Foliar (DAF) para aplicação no software ENVI-met. E\&S - Engineering and Science, 2019, 8:2.

O valor de DAF para cada camada da vegetação bem como padrão de densidade foliar ao longo da altura derivados da Tabela 03 podem ser utilizados para modelar o banco de dados da vegetação utilizada na simulação pelo programa ENVI-met. Já os dados de altura dos indivíduos arbóreos podem ser incluídos conforme demanda de cada modelagem, considerando a variabilidade observada na área de estudo. Destaca-se que pela metodologia proposta, a DAF ajusta-se de forma proporcional ao longo das dez camadas de discretização da copa, contadas estas a partir da superfície do solo até o topo de cada padrão de vegetação.

\section{CONCLUSÃO}

Por meio da pesquisa desenvolvida foi possível confirmar a viabilidade da utilização da metodologia de análise de fotos hemisféricas para obtenção do IAF com uso de lente olho-depeixe acoplada em dispositivos móveis, o que possibilita sua ampla aplicação pelo baixo investimento financeiro requerido. Destaca-se, no entanto, a necessidade de calibração dos resultados por meio do levantamento com o equipamento ceptômetro para emprego desta metodologia em padrões de vegetação diferentes dos abordados nesta pesquisa.

Os valores finais de Índice de área foliar obtidos nesta pesquisa foram $3,445 \mathrm{~m}^{2} / \mathrm{m}^{2}$ para árvores de baixa densidade, $4,913 \mathrm{~m}^{2} / \mathrm{m}^{2}$ para as de média densidade e $6,286 \mathrm{~m}^{2} / \mathrm{m}^{2}$ para as de alta densidade. Estes valores podem servir de orientação preliminar para a definição de faixas de densidade para a região de estudo: valores de IAF inferior a $3,5 \mathrm{~m}^{2} / \mathrm{m}^{2}$, faixa de baixa densidade, entre $3,5 \mathrm{~m}^{2} / \mathrm{m}^{2}$ a $5,0 \mathrm{~m}^{2} / \mathrm{m}^{2}$, média densidade, e superior a $5,0 \mathrm{~m}^{2} / \mathrm{m}^{2}$, alta densidade.

Os fatores de distribuição médios da Densidade de Área Foliar nas 10 camadas da vegetação determinado no estudo foram de $0 \%, 0 \%, 2,8 \%, 13 \%, 16,9 \%, 17,9 \%, 16,9 \%$, $15,3 \%, 11,7 \%$ e $5,5 \%$, contados do chão até o topo da copa. Caso se queira trabalhar por tipo de densidade (baixa, média e alta), os valores encontrados podem ser obtidos na Tabela 1. Destaca-se que em caso de utilização da metodologia para padrões de geometria e densidade foliar diferenciados dos utilizados neste estudo, os fatores devem ser ajustados de forma a representar com mais fidelidade a distribuição real da densidade observada em campo.

A metodologia descrita neste trabalho possibilita a ampliação do banco de dados de Densidade de Área Foliar que aproxima a biblioteca de vegetação do software ENVI-Met dos padrões encontrados em climas tropicais, ampliando assim a possibilidade de aplicação da modelagem computacional para fins de estudo de clima urbano nessas regiões.

\section{REFERÊNCIAS}

AKBARI, H., POMERANTZ, M., \& TAHA, H. Cool surfaces and shade trees to reduce energy use and improve air quality in urban areas. Solar energy, v. 70, n. 3, p.295-310, 2001. https://doi.org/10.1016/S0038-092X(00)00089-X

AMORIM, R. P. L; PEDROSA, M. L.; CARVALHO, H. J. M. Máscara de obstrução: uso de novas tecnologias que facilitam a verificação da obstrução do céu visível. In: XV Encontro Nacional de Tecnologia do Ambiente Construído, v. 15, 2014, Maceió AL. Anais... Maceió AL: ANTAC, 2015. p. 370-378.

BRUSE, M.; FLEER, H. Simulating surface-plant-air interactions inside urban environments with a three dimensional numerical model. Environmental Modeling \& Software, v. 13, p.373-384, 1998. https://doi.org/10.1016/S1364-8152(98)00042-5 
Rosseti, Karyna, et al.; Proposta metodológica para levantamento e configuração de Densidade de Área Foliar (DAF) para aplicação no software ENVI-met. E\&S - Engineering and Science, 2019, 8:2

CUIABÁ. Prefeitura Municipal de Cuiabá. Perfil socioeconômico de Cuiabá, v. 4. Org. Adriana Bussiki Santos; Coord. Jandira Maria Pedrollo. Cuiabá, MT: Central de Texto, 2010.

FAVARIN, J. L.; NETO, D. D. GARCIA, A.; VILLA NOVA, N. A.; FAVARIN, M. G. G. V. Equações para a estimativa do índice de área foliar do cafeeiro. Pesquisa Agropecuária Brasileira, v. 37, n. 6, p.769-773, 2002. http://dx.doi.org/10.1590/S0100204X2002000600005

GUSSON, C.S.; SHINZATO, P. ENVI-metV4 Preview. Apostila apresentada para a disciplina Conforto Ambiental em Espaços Urbanos Abertos. Faculdade de Arquitetura e Urbanismo da Universidade de São Paulo. São Paulo, 14 abr. 2016. Disponível em $<$ https://docplayer.com.br/84197571-Envi-met-v4-preview-carolina-dos-santos-gusson-paulashinzato.html>. Acesso em 12 mai. 2019.

HONJO, T., \& TAKAKURA, T. Simulation of thermal effects of urban green areas on their surrounding areas. Energy and Buildings, v. 15, p.443-446, 1990. https://doi.org/10.1016/0378-7788(90)90019-F

HOSOI, F., AND OMASA, K. Estimating vertical plant area density profile and growth parameters of a wheat canopy at different growth stages using three-dimensional portable lidar imaging. ISRPS Journal of Photogrammetry and Remote Sensing, v.64, p.151-158. 2009. https://doi.org/10.1016/j.isprsjprs.2008.09.003

HUANG, S., TANIGUCHI, M., YAMANO, M., \& WANG, C. H. Detecting urbanization effects on surface and subsurface thermal environment - a case study of Osaka. Science of the $\begin{array}{lllll}\text { total environment, } & \text { v. } & \text { 407, } & \end{array}$ https://doi.org/10.1016/j.scitotenv.2008.04.019

MACHADO, N. G.; BIUDES, M. S.; QUERINO, C. A. S.; DANELICHEN, V. H. de M.; VELASQUE, M. C. S. Seasonal and interannual pattern of meteorological variables in Cuiabá, Mato Grosso State, Brazil. Revista Brasileira de Geofísica, v. 33, p.1-10, 2015. http://dx.doi.org/10.22564/rbgf.v33i3.949

MIRANDA, S. A., DE PAULA, D. C. J., DE MUSIS, C. R., DE SOUZA NOGUEIRA, J., DE MOURA SANTOS, F. M., NOGUEIRA, M. C. J. A. Análise de proposta alternativa na obtenção do fator de visão de céu por método fotográfico. Revista Brasileira de Climatologia, v. 23, p.88-105, 2018. http://dx.doi.org/10.5380/abclima.v23i0.48251

NAKATA, C. M. Comportamento do pedestre e ambiente térmico urbano. 2010. Dissertação (Mestrado). 132f. Pós-Graduação em Design, Universidade Estadual Paulista "Julio de Mesquita Filho", Bauru. 2010.

NAKATA-OSAKI, C. M.; SOUZA, L. C. L. de; RODRIGUES, D. S. Impacto da geometria do cânion urbano na intensidade de ilha de calor noturna: análise através de um modelo simplificado adaptado a um SIG. Ambiente Construído. v. 16, p.73-87, 2016. http://dx.doi.org/10.1590/s1678-86212016000300093 
Rosseti, Karyna, et al.; Proposta metodológica para levantamento e configuração de Densidade de Área Foliar (DAF) para aplicação no software ENVI-met. E\&S - Engineering and Science, 2019, 8:2.

NASCIMENTO, A.R.T.; FAGG, J.M.F.; FAGG, C.W. Canopy openness and LAI estimates in two seasonally Deciduous forests on limestone outcrops in central Brazil using hemispherical photographs. Rev. Árvore, v. 31, p.167-176, 2007. http://dx.doi.org/10.1590/S0100-67622007000100019

OKE, T. R. The energetic basis of the urban heat island. Quarterly Journal of the Royal Meteorological Society, v. 108, n. 455, p.1-24, 1982. https://doi.org/10.1002/qj.49710845502

ROsseti, K. A. C., DURAnTe, L. C., NOGUEIRA, M. C. J. A., CAlleJAS, I. J. A., KUHNEN, I. A., NOGUEIRA, J. S. Efeitos da incorporação de vegetação em telhados de zona urbana em clima tropical continental. PARC Pesquisa em Arquitetura e Construção, v. 6, p.121-128, 2015. https://doi.org/10.20396/parc.v6i2.8635011

SHINZATO, Paula. O impacto da vegetação nos microclimas urbanos. 2009. Dissertação (Mestrado). 173f. Pós-Graduação em Arquitetura e Urbanismo, Universidade de São Paulo, 2009.

SOUZA, C. L.; PONZONI, F. J. Relação entre índice de área foliar, estimado através de sensoriamento remoto, e parâmetros dendrométricos em floresta implantada de Pinus spp. In: IX SIMPÓSIO BRASILEIRO DE SENSORIAMENTO REMOTO, v.9, 1998, Santos. Anais... Santos: INPE/SELPER, 1998. CD-Rom.

SPANGENBERG, J., SHINZATO, P., JOHANSSON, E., \& DUARTE, D. Simulation of the influence of vegetation on microclimate and thermal comfort in the city of São Paulo. Revista da Sociedade Brasileira de Arborização Urbana, v. 3, p.1-19, 2019. http://dx.doi.org/10.5380/revsbau.v3i2.66265

UPMANIS, H., ELIASSON, I., LINDQVIST, S. The influence of green areas on nocturnal temperatures in a high latitude city (Göteborg, Sweden). International journal of climatology, v. 18, n. 6, p.681-700, 1998.

WATSON, D. J. The physiological basis of variation in yield. Advances in Agronomy, v. 4, p.101-144, 1952. https://doi.org/10.1016/S0065-2113(08)60307-7 